\title{
MAMMALIAN AND AVIAN DIVERSITY OF THE NALLAMALA HILLS, ANDHRA PRADESH
}

\author{
C. Srinivasulu and V. Nagulu
}

Wildlife Biology Section, Department of Zoology, Osmania University, Hyderabad, Andhra Pradesh 500007, India

Email:masawa@hd2.dot.net.in

\begin{abstract}
The paper briefly reviews the mammalian and avian diversity of the Nallamala Hills $\left(15^{\circ} 20^{\prime}-16^{\circ} 31^{\prime} \mathrm{N}\right.$ and $\left.78^{\circ} 30^{\prime}-80^{\circ} 10^{\prime} \mathrm{E}\right)$, Andhra Pradesh. Based on faunal surveys carried out intermittently throughout the study area and published information, the presence of 74 mammals and 302 bird species in this stretch of Eastern Ghats is reported.
\end{abstract}

\section{Keywords}

Diversity, mammals, birds, Nallamala Hills, Eastern Ghats, Andhra Pradesh

\section{Introduction}

Nallamala Hills stretching across five districts of the state of Andhra Pradesh is renowned for being rich in biodiversity (Rao, 1998). The first of the faunal surveys conducted here dates back to 1930, when Dr. Sàlim Ali had collected bird specimens from Mananur and Farahabad on the Amrabad Plateau of these hills during the Hyderabad State Ornithological Survey (Ali 1933a, b, c; 1934a, b). From avian point of view further surveys were carried out by members of the Birdwatchers Society of Andhra Pradesh (Aasheesh Pittie, pers. comm.), and the research team at the Zoology Department of Osmania University (Rao et al., 1997; Srinivasulu \& Rao, 1999). Excepting a few faunal surveys carried out by Zoological Survey of India (Murthy, 1968, 1986; Sharma, 1971; Agrawal \& Bhattacharya, 1976) and by the Forest Department, no scientific documentation of mammalian and avian fauna was done until recently. Nagulu et al. (1998) gave an account of these in the context of Eastern Ghats in general.

We have been regularly monitoring the mammalian and avian diversity of the Nallamala Hills, especially along the tracts encompassed by Nagarjunasagar Srisailam Tiger Reserve (3568 $\mathrm{km}^{2}$ ) and Gundla Brahmeshwaram Wildlife Sanctuary $\left(1198 \mathrm{~km}^{2}\right)$.
Intermittent faunal surveys in the Nallamala Hills have been an on going task since 1990 by researchers of our department. The senior author had carried out systematic studies on mammalian and avifaunal diversity between March 1996 to November 2000, while studying ungulate behaviour for his doctoral research. Published documents, and information gathered from officials of the Project Tiger, Forest Department and tribals (Chenchus) has also been relied upon. This article briefly reviews the mammalian and avian diversity of the Nallamala Hills.

\section{Study area}

Nallamala $\left(15^{\circ} 20^{\prime}-16^{\circ} 31^{\prime} \mathrm{N}\right.$ and $\left.78^{\circ} 30^{\prime}-80^{\circ} 10^{\prime} \mathrm{E}\right)$ is a group of low hill ranges in the central part of Eastern Ghats (Fig. 1). From the Palnad basin in the north to the Tirupati basin in the south, the Nallamala runs a distance of $430 \mathrm{~km}$ with an average width of $30 \mathrm{~km}$ (Anon, 1965). An unbroken chain of rugged hills with precipitous cliffs encompassing an area of about $7640 \mathrm{~km}^{2}$ forms the range. The vegetation is typically of southern tropical dry deciduous and southern tropical moist deciduous forest types intermingled with scrub (Champion \& Seth, 1968). The climate is generally hot and dry with temperatures rising up to $43^{\circ} \mathrm{C}$ to $45^{\circ} \mathrm{C}$ during May and dips down to $8^{\circ} \mathrm{C}$ in December. Average rainfall in this region is between 900 and $1000 \mathrm{~mm}$.

\section{Methodology}

Mammalian fauna was recorded either by direct sightings or by the presence of indirect evidence. Footprints, droppings, and kills (both by predator and road) were carefully analysed. Mammals were categorised to be common (fairly well distributed and sighted, or evidence recorded once a day), uncommon (fairly well distributed and sighted, or evidence recorded once a week), occasional (restricted distribution and sighted, or evidence recorded infrequently), and rare (fewer than 10 sightings, or evidence recorded, or single sight records either by us, or reliable sources).

Survey of birds was carried regularly from March 1996 to 


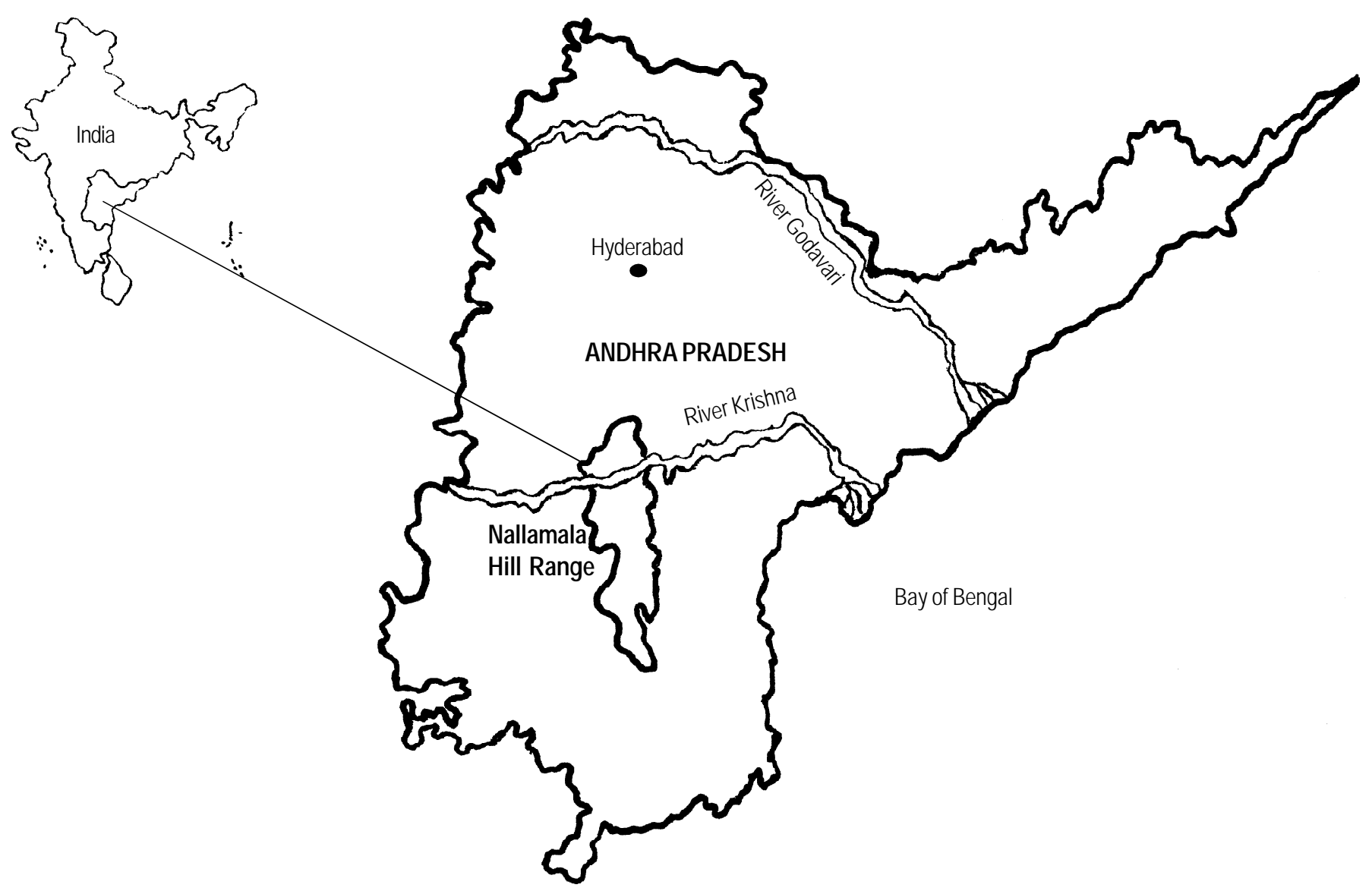

Figure 1. Map depicting the Nallamala Hill Range in Andhra Pradesh, India

February 2000, to ensure that all months of the year were covered. Bird records were done following imaginary grid method and line transect method as outlined by Gaston (1973). Identification was based on Ali and Ripley (1983) and Ali (1996). Based on the number of sightings and occurrence of a given species, their status was assigned. Birds were considered to be common (encountered daily in relatively large numbers), uncommon (encountered daily but in few numbers), occasional (encountered occasionally in less numbers, or singly) or rare (encountered less than 15 times in a year). Scientific names and taxonomic classification of birds is after Inskipp et al. (1996) and common names after Manakadan et al. (1998).

\section{Results and Discussion}

The mammalian diversity of this region is represented by 74 species belonging to 28 families of nine orders (Table 1). Maximum number of species recorded or reported to occur in the Nallamala Hills belonged to order Chiroptera (24 species) and was followed by orders Carnivora (20 species) and Rodentia (12 species). The known rodents from this area being so less in number calls for collection of specimens in future to have a complete picture of the small mammalian diversity here. The Nallamala mammals represent about $20 \%$ of the Indian and $2 \%$ of the world mammalian diversity.

Of the total mammalian diversity recorded to occur here, $55.4 \%$ (41 species) are common, 27.02\% (20 species) are uncommon, $12.16 \%$ ( 9 species) are occasional and 5.4\% (4 species) are rare in occurrence. Among the mammalian species reported by us in the Nallamala Hills, 69 species $(93.24 \%)$ were sighted during the study period, and about $4.3 \%$ (3 species) are based on indirect evidence. The presence of five species $(6.75 \%)$ of mammals that were not recorded by us during the present study, is reported on account of reliable information. These interesting mammalian records are: Madras Hedgehog Hemiechinus nudiventris, Wolf Canis lupus, Indian Pangolin Manis crassicaudata; Asiatic Wild Cat Felis sylvestris ornata and Indian Muntjak Muntiacus muntjac (Tulsi Rao, pers. comm.).

Some of the infrequently sighted species that deserve mention 
are the Smooth-coated Otter Lutrogale perspicillata (along the selected stretch of Krishna River), Ratel Mellivora capensis (more on the south of bank of Krishna River along the Pecheruvu and Gundla Brahmeshwaram Plateaus), and Fishing Cat Prionailurus viverrinus (recorded once on the banks of Gundlakamma River on the Gundla Brahmeshwaram Plateau). The Chinkara Gazella bennetti is found in the open tracts along the Krishna River on the Amrabad Plateau, while the Blackbuck Antilope cervicapra is reported only from Ganjivaripalle environs.

The species of the monotypic genera of mammals occurring in the Nallamala are Indian Tree Shrew Anathana ellioti (Scadentia), Indian Wild Dog Cuon alpinus, Sloth Bear Melursus ursinus, Ratel, Hyena Hyaena hyaena, Small Indian Civet Vivvericula indica (Carnivora), Blackbuck, Nilgai Boselaphus tragocamelus, Four-horned Antelope Tetracerus quadricornis (Artiodactyla) and Indian Bush Rat Golunda ellioti (Rodentia). Of the threatened species, the endangered ones that are found in the Nallamala are Tiger Panthera tigris, Ratel, Rusty-spotted Cat Prionailurus rubiginosus (Rao et al., 1999), and Four-horned Antelope Tetracerus quadricornis, which is densely populated on the Gundla Brahmeshwaram Plateau. The species that are endemic to the peninsular India and are thriving on the Nallamala Hills are Indian Tree Shrew (Scadentia), Bonnet Macaque Macaca radiata (Primates), and Giant Squirrel Ratufa indica (Rodentia).

Complete representation of smaller mammals occurring in the Nallamala Hills might have not been covered in the present study. As a result many smaller forms, especially those belonging to orders Insectivora, Chiroptera and Rodentia are poorly represented in this study. Careful scrutiny of the relevant published material revealed the historic presence of many species that were not reported in the recent times. Among the chiropterans, Lesser Woolly Horseshoe Bat Rhinopoma beddomei and Andersen's Leaf-nosed Bat Hipposideros pomona are such examples which were earlier collected from the Nallamala Hills (Bates \& Harrison, 1996) but the present day status remains unknown.

The avian diversity of the Nallamala Hills is represented by a total of 302 species belonging to 17 orders and 53 families (Table 2). Passerines represented by 125 species belonging to 20 families contribute to about $41.4 \%$ of the bird diversity. Among the non-passerines maximum representation were that of waders (Charadriiformes, 6 families and 29 species) and diurnal raptors (Falconiformes, 2 families, 26 species). Other orders represented in significant numbers of species are Anseriformes ( 2 family, 15 species), Ciconiiformes (3 families, 17 species), Gruiiformes (3 families, 14 species), Coraciiformes ( 7 families, 12 species) Galliformes (1 family, 11 species), and Columbiformes ( 2 families, 11 species).
Of the total diversity, $48.67 \%$ (147 species) are common, $32.45 \%$ (98 species) are uncommon, $12.25 \%$ (37 species) are occasional, and $6.62 \%$ (20 species) are rare in abundance. Of the resident bird diversity of 189 species, $91.53 \%$ are confirmed residents, while the rest are resident migrants. Among the migrants represented by 101 species (contributing $33.44 \%$ of the total diversity) most are winter migrants (73 species, $72.27 \%$ ) followed by seasonal local migrants (23 species, 22.77\%) and local migrants (5 species, $4.95 \%$ ). Twelve species (3.97\% of the total diversity) are considered to be vagrant or stragglers to this area. Of the total diversity 136 species $(45.03 \%)$ are confirmed breeders while about 41 species (13.57\%) probably breed here.

Ali (1933a, b, c; 1934a, b) has reported sighting of 127 species of birds on Amrabad Plateau from Mananur and Farahabad localities between 3-20 October, 1931, of which 123 birds were recorded during the present study. Species reported by Ali that were conspicuously absent here and elsewhere in the Nallamala Hills are Eurasian Griffon-Vulture Gyps fulvus (reported to be common then), Indian Trogon Harpactes fasciatus (one sighted on 12 October, 1931 on Uma Maheshwara Plateau near Mananur), Nilgiri Wood Pigeon Columba elphinstonii (Ali, (1934b) mentions catching a glimpse of it in the forest around Mananur), and Greenish Warbler (Phylloscopus trochiloides nitidus and $P$. $t$. viridanus). It is possible that the last species might have been possibly overlooked or confused with other species of Phylloscopus. Some of the interesting records of the present study are reporting of sighting of White-necked Stork Ciconia episcopus, White Stork Ciconia ciconia along the Madras Canal near Veligode, Pied Avocet Recurvirostra avosetta on the Amrabad Plateau (Tulsi Rao, pers. comm.), Indian Pied Hornbill Anthracoceros coronatus near Peddarutla on the Right Bank of Nagarjunasagar Srisailam Tiger Reserve (Harsha Reddy, pers. comm.), Yellow-throated Bulbul near Uma Maheshawaram (Aasheesh Pittie, pers. comm.) and from two other sites, namely Rollapenta and Pedda Manthanala on the right bank of Nagarjunasagar Srisailam Tiger Reserve (Srinivasulu in prep.), Yellow-browed Bulbul from Uma Maheshwaram (Srinivasulu \& Rao, 2000), Little Pied Flycatcher Ficedula westermanni from Gundla Brahmeshwaram Plateau (Srinivasulu, in press), and White-rumped Shama Copsychus malabaricus from Farahabad on Amrabad Plateau (Rao et al., 1997) and Rudrakode area of Gundla Brahmeshwaram Wildlife Sanctuary .

The species of the monotypic genera of birds occurring in the Nallamala are Anastomus oscitans (Ciconiformes), Rhodonessa rufina (Anseriformes), Butastus teesa (Falconiformes), Hydrophasianus chirurgus and Metopidius indicus (Charadriiformes). 


\section{Threats}

The once dense and secure forest of Nallamala is under severe threat due to habitat destruction, livestock pressure and biotic interference. Human greed has taken its toll and the aftereffects of the same is reflected by the deserted look of the forest, especially across the Amrabad plateau. Rapid developmental activities are underway jeopardizing biotic integrity and sustenance of floral and faunal components. The northern part of the Nallamala Hills, especially that north of Krishna River falling in the districts of Mahbubnagar and Nalgonda, has been greatly affected by anthropogenic and biotic pressure, while the central and southern regions are relatively better. Management intervention should ensure checking of illegal entry, livestock pressure, poaching, tree felling, etc. to ensure conservation priorities and sustenance of sylvan tribal population. The treasure-chest will soon be empty without timely conservation measures.

\section{Acknowledgements}

We express our heartfelt gratitude Prof. J.V. Ramana Rao for guidance and encouragement. We are thankful to the Head Department of Zoology, Osmania University, Hyderabad and Shri T. Ramakrishna, IFS, Chief Wildlife Warden and Conservator of Forests (Wildlife Management), Andhra Pradesh Forest Department for permission. We thank immensely, Field Directors Project Tiger; Assistant Directors NSTR; Mr. K. Tulsi Rao (ACF, Biodiversity, NSTR); Mr. Aasheesh Pittie; Dr. V. Vasudeva Rao; Mr. Harsha Reddy for their help in many ways and sharing information. We acknowledge the financial assistance by the Council for Scientific and Industrial Research, New Delhi to the first author and, the Andhra Pradesh Forest Department (A. P. Forestry Project), Government of Andhra Pradesh to the latter.

\section{References}

Agrawal, V.C. and T. P. Bhattacharya (1976). Report on a collection of mammals from Nagarjunasagar, Andhra Pradesh. Newsletter of the Zoological Survey of India 2(5): 212-216.

Ali, S. (1933a). The Hyderabad State Ornithological Survey. Part 1. Journal of the Bombay Natural History Society 36(2): 356-390.

Ali, S. (1933b). The Hyderabad State Ornithological Survey. Part 2. Journal of the Bombay Natural History Society 36(3): 707-725.

Ali, S. (1933c). The Hyderabad State Ornithological Survey. Part 3. Journal of the Bombay Natural History Society 36(4): 898-919.

Ali, S. (1934a). The Hyderabad State Ornithological Survey. Part 4. Journal of the Bombay Natural History Society 37(1): 124-142.

Ali, S. (1934b). The Hyderabad State Ornithological Survey. Part 5. Journal of the Bombay Natural History Society37(2): 425-454.

Ali, S. (1996). The Book of Indian Birds. Revised and Enlarged Edition. The Bombay Natural History Society and Oxford University Press, Mumbai.

Ali, S. and S.D. Ripley (1983). A Pictorial Guide to the Birds of the Indian Subcontinent. Bombay Natural History Society and Oxford University Press, Mumbai.

Anon. (1965). The Gazetteer of India - Indian Union. Vol. I. Country and People. Publications Division, Govt. of India, New Delhi.

Bates, P.J.J. and D.L. Harrison (1997). Bats of the Indian Subcontinent. Harrison Zoological Museum, Kent, xiv+358pp.

Champion, H.G. and S.K. Seth (1968). A revised survey of Forest types of India. Govt. of India, New Delhi.

Gaston, A.J. (1973). Methods for estimating bird populations. Journal Bombay Natural History Society 72(2): 272-281.

Inskipp, T., N. Lindsey and W. Duckworth (1996). An Annotated Checklist of the Birds of the Oriental Region. Oriental Bird Club, United Kingdom.

Manakadan, R., J.C. Daniel, A.R. Rahmani, M. Inamdar and G. Ugra (1998). Standardized English common names of the birds of the Indian sub continent - a proposal. Buceros 3(2): 55.

Murthy, T.S.N. (1968). Notes on the collection of amphibians from Nagarjuna Valley (Andhra Pradesh) with one new record. Journal of the University of Poona 34: 63-71.

Murthy, T.S.N. (1986). Lizards of Kurnool District. Bulletin of Maryland Herpetological Society 22(3): 134-143.

Nagulu, V., V.V. Rao and C. Srinivasulu (1998). Biodiversity of select habitats in Eastern Ghat regions of Andhra Pradesh. In: The Eastern Ghats Proceedings of the National Seminar on Conservation of Eastern Ghats March 24-26, 1998. 6-35.

Nameer. P.O. (1998). Checklist of Indian Mammals. Kerala Forest Department (Wildlife Wing) and Kerala Agricultural University. 90+xxvpp.

Rao, R.K. (1998). Nallamalai Hills: among world centres of plant diversity. In: The Eastern Ghats Proceedings of the National Seminar on Conservation of Eastern Ghats, March 24-26, 1998. 317-321.

Rao, T.K., D. Sudhakar, V.V. Rao, V. Nagulu and C. Srinivasulu (1999). Rusty-spotted Cat Prionailurus rubiginosus (I. Geoffroy Saint-Hillaire, 1831): A new record for Nagarjunasagar Srisailam Tiger Reserve, Andhra Pradesh. Journal of the Bombay Natural History Society 96(3): 463-464.

Rao, V.V., V. Nagulu, M. Anjaneyulu, B. Srinivasulu, C. Srinivasulu and J. V. R. Rao (1997). Status of avifauna of Rajiv Gandhi National Park, Andhra Pradesh, India. Pavo 35(1\&2): 85100.

Ripley, S.D. (1982). A Synopsis of Birds of India and Pakistan. Bombay Natural History Society, Bombay.

Sharma, R.C. (1971). The reptile fauna of Nagarjunasagar dam area (Andhra Pradesh, India). Records of the Zoological Survey of India 63(1-4): 77-93.

Srinivasulu, C. (in prep.). Site records of Yellow-throated Bulbul Pycnonotus xantholaemus in the Nallamala Hills, Eastern Ghats, Andhra Pradesh.

Srinivasulu, C. (in press). Sight record of Little Pied Flycatcher Muscicapa (Ficedula) westermanni in Andhra Pradesh. Journal of the Bombay Natural History Society.

Srinivasulu, C. and V.V. Rao (1999). Butterflies of Gundla Brahmeshwaram Wildlife Sanctuary, Andhra Pradesh. Zoos' Print 14(2): 38-39.

Srinivasulu, C. and V.V. Rao (2000). Occurrence of the Yellowbrowed Bulbul Hypsipetes indicus (Jerdon) in the Nalamalla Hills, Andhra Pradesh. Journal of the Bombay Natural History Society 97(1): 144-145. 
Table 1. Mammalian diversity of the Nallamala Hills, Eastern Ghats

\begin{tabular}{|c|c|c|c|c|c|c|c|}
\hline Common name & Scientific name & A & $\mathbf{R}$ & Common name & Scientific name & A & $\mathbf{R}$ \\
\hline $\begin{array}{l}\text { Insectivora } \\
\text { Erinaceidae }\end{array}$ & & & & $\begin{array}{l}\text { Carnivora } \\
\text { Canidae }\end{array}$ & & & \\
\hline$\overline{\text { Madras Hedgehog }}$ & Hemiechinus nudiventris & $\mathrm{R}$ & 3 & $\overline{\text { Golden Jackal }}$ & Canis aureus & $\mathrm{C}$ & 1 \\
\hline Soricidae & & & & Wolf & Canis lupus & 0 & 3 \\
\hline$\frac{\text { Dulcuate }}{\text { Musk Rat }}$ & Suncus murinus & C & 1 & $\begin{array}{l}\text { Dhole Indian Wild Dog } \\
\text { Bengal (Indian) Fox }\end{array}$ & $\begin{array}{l}\text { Cuon alpinus } \\
\text { Vulpes bengalensis }\end{array}$ & $\begin{array}{l}\mathrm{C} \\
\mathrm{C}\end{array}$ & $\begin{array}{l}1 \\
1\end{array}$ \\
\hline $\begin{array}{l}\text { Scadentia } \\
\text { Tupaiidae }\end{array}$ & & & & $\underline{\text { Ursidae }}$ & & & \\
\hline $\begin{array}{l}\text { Iupalldad } \\
\text { South Indian (Madras) Tree } \\
\text { Shrew }\end{array}$ & Anathana ellioti & UC & 1 & $\begin{array}{l}\text { Sloth Bear } \\
\text { Mustelidae }\end{array}$ & Melursus ursinus & $\mathrm{C}$ & 1 \\
\hline $\begin{array}{l}\text { Chiroptera } \\
\text { Pteropodidae }\end{array}$ & & & & $\begin{array}{l}\text { Smooth-coated Otter } \\
\text { Ratel (Honey Badger) }\end{array}$ & $\begin{array}{l}\text { Lutrogale perspicillata } \\
\text { Mellivora capensis }\end{array}$ & $\begin{array}{l}\text { UC } \\
\text { UC }\end{array}$ & $\begin{array}{l}1,3 \\
1,3\end{array}$ \\
\hline Fulvous Fruit Bat & Rousettus leschnaulti & UC & 1 & $\underline{\text { Viverridae }}$ & & & \\
\hline Indian Flying Fox & Pteropus giganteus & UC & 1,2 & $\overline{\text { Common Palm Civet }}$ & Paradoxurus hermaphroditus & $\mathrm{C}$ & 1 \\
\hline Short-nosed Fruit Bat & Cynopterus sphinx & C & 1 & Small Indian Civet & Viverricula indica & C & 1 \\
\hline Rhinopomatidae & & & & Hespertidae & & & \\
\hline Lesser Mouse-tailed Bat & Rhinopoma hardwickii & UC & 1,2 & $\overline{\text { Grey (Common) Mongoose }}$ & Herpestes edwardsii & C & 1 \\
\hline Emballonuridae & & & & Small Indian Mongoose & Herpestes javanicus & C & 1 \\
\hline Long-winged Tomb Bat & Taphozous longimanus & UC & 1,2 & Ruddy Mongoose & Herpestes smithii & C & 1 \\
\hline Black-bearded Tomb Bat & Taphozous melanopogon & UC & 1,2 & $\underline{\text { Hyaenidae }}$ & & & \\
\hline Naked-rumped Tomb Bat & Taphozous nudiventris & 0 & 1 & Striped Hyaena & Hyaena hyaena & 0 & 1 \\
\hline Egyptian Tomb Bat & Taphozous perforatus & 0 & 1 & Felidae & & & \\
\hline Megadermatidae & & & & $\overline{\text { Jungle Cat }}$ & Felis chaus & $\mathrm{C}$ & 1 \\
\hline$\overline{\text { Greater False Vampire }}$ & Megadermalyra & C & 1,2 & Desert (Wild) Cat & Felis silvestris & $\mathrm{R}$ & 3 \\
\hline Lesser False Vampire & Megaderma spasma & 0 & 1 & Leopard Cat & Prionailurus bengalensis & C & 1 \\
\hline Rhinolophidae & & & & Rusty-spotted Cat & Prionailurus rubiginosus & $\mathrm{R}$ & 1 \\
\hline Rufous Horseshoe Bat & Rhinolophus rouxii & C & 1,2 & $\begin{array}{l}\text { Fishing Cat } \\
\text { Leopard }\end{array}$ & $\begin{array}{l}\text { Prionailurus viverrinus } \\
\text { Panthera pardus }\end{array}$ & $\begin{array}{l}\mathrm{O} \\
\mathrm{C}\end{array}$ & $\begin{array}{l}1,3 \\
1\end{array}$ \\
\hline Hipposideridae & & & & Tiger & Panthera tigris & C & 1 \\
\hline Fulvus Leaf-nosed Bat & Hipposideros fulvus & 0 & 1 & Artiodactyla & & & \\
\hline Kelaart's Leaf-nosed Bat & Hipposideros lankadiva & 0 & 1 & Suidae & & & \\
\hline $\begin{array}{l}\text { Schneider's Leaf-nosed Bat } \\
\text { Molossidae }\end{array}$ & Hipposideros speoris & UC & 1,2 & Wild Boar & Sus scrofa cristatus & C & 1 \\
\hline $\begin{array}{l}\text { Egyptian Free-tailed Bat } \\
\text { Vespertilionidae }\end{array}$ & Tadarida aegyptiaca & C & 1 & $\frac{\text { Tragulidae }}{\text { Indian Chevrotain }}$ & Moschiola meminna & C & 1 \\
\hline Tickell's Bat & Hesperoptenus tickelli & UC & 1 & Cervidae & & & \\
\hline Painted Bat & Kerivoula picta & UC & 1 & Spotted Deer & Axis axis & C & 1 \\
\hline Schreiber's Long-fingered Bat & Miniopterus schreibersii & UC & 1 & Sambar & Cervus unicolor & $\mathrm{C}$ & 1 \\
\hline Kelaart's Pipistrelle & Pipistrellus ceylonicus & $\mathrm{C}$ & 1 & Indian Muntjak & Muntiacus muntjak & UC & 3 \\
\hline Little Indian Bat & Pipistrellus coromandra & $\mathrm{C}$ & 1 & Bovidae & & & \\
\hline Dormer's Bat & Pipistrellus dormeri & $\mathrm{R}$ & 1 & $\overline{\text { Blackbuck }}$ & Antilope cervicapra & UC & 1 \\
\hline Least Pipistrelle & Pipistrellus tenuis & $\mathrm{C}$ & 1,2 & Bluebull & Boselaphus tragocamelus & $\mathrm{C}$ & 1 \\
\hline Asiatic Greater Yellow House & Scotophillus heathii & UC & 1,2 & Indian Gazelle & Gazella bennetti & UC & 1 \\
\hline Bat & & & & Four-horned Antelope & Tetracerus quadricornis & UC & 1 \\
\hline $\begin{array}{l}\text { Asiatic Lesser Yellow House } \\
\text { Bat }\end{array}$ & Scotophillus kuhlii & UC & 1,2 & $\begin{array}{l}\text { Pholidota } \\
\text { Manidae }\end{array}$ & & & \\
\hline Primates & & & & $\overline{\text { Indian Pangolin }}$ & Manis crassicaudata & 0 & 3 \\
\hline Cercopithecidae & & & & Rodentia & & & \\
\hline$\overline{\text { Rhesus Macaque }}$ & Macaca mulatta & C & 1 & $\begin{array}{l}\text { Rodentla } \\
\text { Sciuridae }\end{array}$ & & & \\
\hline Bonnet Macaque & Macaca radiata & C & 1 & Indian (Three-striped) Palm & Funambulus palmarum & C & 1 \\
\hline Common (Hanuman) Langur & Semnopithecus entellus & C & 1 & $\begin{array}{l}\text { Squirrel } \\
\text { Northern (Five-striped) Palm }\end{array}$ & Funambulus pennantii & C & 1 \\
\hline
\end{tabular}




\begin{tabular}{|c|c|c|c|}
\hline Common name & Scientific name & Abn & Rem \\
\hline \multicolumn{4}{|l|}{ Squirrel } \\
\hline Indian Giant Squirrel & Ratufa indica & C & 1 \\
\hline \multicolumn{4}{|l|}{ Muridae } \\
\hline$\overline{\text { Indian Gerbill }}$ & Tatera indica & UC & 1 \\
\hline Indian Bush Rat & Golunda ellioti & $\mathrm{C}$ & 1 \\
\hline Soft-furred Field Rat & Millardia meltada & C & 1 \\
\hline Brown Rat & Rattus norvegicus & C & 1 \\
\hline House Rat & Rattus rattus & $\mathrm{C}$ & 1 \\
\hline House Mouse & Mus musculus & $\mathrm{C}$ & 1 \\
\hline Little Indian Field Mouse & Mus booduga & $\mathrm{C}$ & 1 \\
\hline Lesser Bandicoot-rat & Bandicota bengalensis & C & 1 \\
\hline Greater Bandicoot-rat & Bandicota indica & UC & 1 \\
\hline \multicolumn{4}{|l|}{ Hystricidae } \\
\hline Indian Crested Porcupine & Hystrix indica & C & 1 \\
\hline \multicolumn{4}{|l|}{ Lagomorpha } \\
\hline \multicolumn{4}{|l|}{ Leporidae } \\
\hline Indian Hare & Lepus nigricollis & $\mathrm{C}$ & 1 \\
\hline
\end{tabular}

Abn- Abundance; Rem - Remarks

C - Common; UC - Uncommon; O - Occasional; R - Rare;

1 - Species sighted or recorded to occur in Nallamala Hills during the study;

1,2 - Species that were collected or recorded by earlier workers (especially the chiropterans); 1, 3 - Species that were reported to occur, not sighted during the present survey, but indirect evidence observed; 3 -Species reported to occur by reliable source, not recorded during the present study.

Common names also follow Nameer (1998).
Table 2. Avifaunal diversity of the Nallamala Hills, Eastern Ghats

\begin{tabular}{|c|c|c|c|}
\hline Common name & Scientific name & Abn & Status \\
\hline \multicolumn{4}{|l|}{$\begin{array}{l}\text { Podicipitiformes } \\
\text { Podicpedidae }\end{array}$} \\
\hline Little Grebe & Tachybaptus ruficollis* (5) & C & $\mathrm{Res}, \mathrm{Br}$ \\
\hline \multicolumn{4}{|l|}{ Pelecaniformes } \\
\hline Great Cormorant & Phalacrocorax carbo (26) & $\mathrm{R}$ & SLM \\
\hline Indian Cormorant & Phalacrocorax fuscicollis (27) & 0 & SLM \\
\hline Little Cormorant & Phalacrocorax niger (28) & UC & $\mathrm{Res}, \mathrm{Br}$ \\
\hline Darter & Anhinga rufa (29) & 0 & SLM \\
\hline \multicolumn{4}{|l|}{$\begin{array}{l}\text { Ciconiiformes } \\
\text { Ardeidae }\end{array}$} \\
\hline Grey Heron & Ardea cinerea (35) & C & $\mathrm{LM}, \mathrm{Br}$ \\
\hline Purple Heron & Ardea purpurea (37) & $\mathrm{C}$ & $\mathrm{LM}, \mathrm{Br}$ ? \\
\hline Indian Pond-Heron & Ardeola grayili $^{*}(42)$ & $\mathrm{C}$ & $\mathrm{Res}, \mathrm{Br}$ \\
\hline Cattle Egret & Bubulcus ibis (44) & C & $\mathrm{Res}, \mathrm{Br}$ \\
\hline Great Egret & Casmerodius albus (45) & UC & Res? \\
\hline Intermediate Egret & Mesophoyx intermedia (47) & $\mathrm{C}$ & Res? \\
\hline Little Egret & Egretta garzetta (49) & C & $\mathrm{Res}, \mathrm{Br}$ \\
\hline $\begin{array}{l}\text { Black-crowned Night- } \\
\text { Heron }\end{array}$ & Nycticorax nycticorax (52) & $\mathrm{C}$ & Res, $\mathrm{Br}$ \\
\hline Cinnamon Bittern & Ixobrychus cinnamomeus (56) & UC & $\mathrm{Res}, \mathrm{Br}$ ? \\
\hline Yellow Bittern & Ixobrychus sinensis (57) & UC & $\mathrm{Res}, \mathrm{Br}$ ? \\
\hline \multicolumn{4}{|l|}{ Ciconiidae } \\
\hline Painted Stork & Mycteria leucocephala (60) & $\mathrm{C}$ & SLM \\
\hline Asian Open-billed Stork & Anastomus oscitans (61) & $\mathrm{C}$ & $\mathrm{Res}, \mathrm{Br}$ \\
\hline White-necked Stork & Ciconia episcopus (62) & 0 & SLM \\
\hline White Stork & Ciconia ciconia (63) & 0 & WM \\
\hline \multicolumn{4}{|l|}{ Threskiornithidae } \\
\hline$\overline{\text { Asian White Ibis }}$ & Threskiornis melanocephalus* & 0 & SLM \\
\hline
\end{tabular}

Black Ibis

Eurasian Spoonbill

(69)

$\begin{array}{lll}\text { Pseudibis papillosa (70) } & \text { UC } & \text { Res, Br? } \\ \text { Platalea leucorodia(72) } & \text { UC } & \text { SLM }\end{array}$

$\begin{array}{llll}\begin{array}{l}\text { Anseriformes } \\ \text { Anatidae }\end{array} & & & \\ \text { Bar-headed Goose } & \text { Anser indicus (82) } & \text { O } & \text { WM } \\ \text { Ruddy Shelduck } & \text { Tadorna ferruginea (90) } & \text { UC } & \text { WM } \\ \text { Northern Pintail } & \text { Anas acuta (93) } & \text { C } & \text { WM } \\ \text { Common Teal } & \text { Anas crecca (94) } & \text { C } & \text { WM } \\ \text { Spot-billed Duck } & \text { Anas poecilorhyncha*(97) } & \text { C } & \text { Res, Br } \\ \text { Gadwall } & \text { Anas strepera (101) } & \text { UC } & \text { WM } \\ \text { Eurasian Wigeon } & \text { Anas penelope (103) } & \text { O } & \text { WM } \\ \text { Garganey } & \text { Anas querquedula (104) } & \text { UC } & \text { WM } \\ \text { Northern Shoveller } & \text { Anas clypeata (105) } & \text { UC } & \text { WM } \\ \text { Red-crested Pochard } & \text { Rhodonessa rufina(107) } & \text { UC } & \text { WM } \\ \text { Common Pochard } & \text { Aythya ferina (108) } & \text { UC } & \text { WM } \\ \text { Tutted Pochard } & \text { Aythya fuligula(111) } & \text { R } & \text { WM } \\ \text { Cotton Pygmy-Goose } & \text { Nettapus coromandelianus } & \text { C } & \text { Res, Br } \\ & \text { (114) } & & \\ \text { Comb Duck } & \text { Sarkidiornis melanotos }{ }^{\star}(115) & \text { O } & \text { SLM } \\ \text { Dendrocygnidae } & & & \\ \text { Lesser Whistling Duck } & \text { Dendrocygnajavanica* (88) } & \text { C } & \text { Res, Br }\end{array}$




\begin{tabular}{|c|c|c|c|c|c|c|c|}
\hline Common name & Scientific name & Abn & Status & Common name & Scientific name & Abn & Status \\
\hline $\begin{array}{l}\text { Falconiformes } \\
\text { Accipitridae }\end{array}$ & & & & $\begin{array}{l}\text { Demoiselle Crane } \\
\text { Rallidae }\end{array}$ & Grus virgo (326) & $\mathrm{R}$ & V \\
\hline Black-shouldered Kite & Elanus caeruleus (124) & C & Res, $\mathrm{Br}$ & Water Rail & Rallus aquaticus (328) & $\mathrm{R}$ & $\mathrm{V}$ \\
\hline Oriental Honey Buzzard & Pernis ptilorhynchus ${ }^{\star}(130)$ & UC & $\mathrm{Res}, \mathrm{Br}$ ? & Baillon's Crake & Porzana pusilla (337) & 0 & WM \\
\hline Black Kite & Milvus migrans ${ }^{\star}(133)$ & C & $\mathrm{Res}, \mathrm{Br}$ & Ruddy-breasted Crake & Porzana fusca (340) & 0 & SLM \\
\hline Brahminy Kite & Haliastur indus* (135) & C & Res, $\mathrm{Br}$ & Brown Crake & Amaurornis akool (342) & 0 & $\mathrm{~V}$ \\
\hline Shikra & Accipiter badius* $(138)$ & C & Res, Br & \multicolumn{2}{|c|}{ White-breasted Waterhen Amaurornis phoenicurus (343) } & $\mathrm{C}$ & Res, $\mathrm{Br}$ \\
\hline Crested Goshawk & Accipiter trivirgatus (144) & $\mathrm{R}$ & V & Watercock & Gallicrex cinerea (346) & $\mathrm{R}$ & Res? \\
\hline Long-legged Buzzard & Buteo rufinus (153) & 0 & V & Common Moorhen & Gallinula chloropus (347) & $\mathrm{C}$ & $\mathrm{Res}, \mathrm{Br}$ \\
\hline White-eyed Buzzard & Butastur teesa* (157) & C & Res, $\mathrm{Br}$ & Purple Swamphen & Porphyrio porphyrio (349) & $\mathrm{C}$ & $\mathrm{Res}, \mathrm{Br}$ \\
\hline $\begin{array}{l}\text { Changeable Hawk-Eagle } \\
\text { Tawny Eagle }\end{array}$ & $\begin{array}{l}\text { Spizaetus cirrhatus (161) } \\
\text { Aquila rapax (168) }\end{array}$ & $\begin{array}{l}C \\
C\end{array}$ & Res, $\mathrm{Br}$ & Common Coot & Fulica atra (350) & $\mathrm{C}$ & $\mathrm{Res}, \mathrm{Br}$ \\
\hline Greater Spotted Eagle & Aquila clanga (170) & $\mathrm{R}$ & $\mathrm{V}$ & \multicolumn{4}{|l|}{ Charadriiformes } \\
\hline $\begin{array}{l}\text { Greater Grey-headed } \\
\text { Fish-Eagle }\end{array}$ & Ichthyophaga icthyaetus (175) & $\mathrm{R}$ & $\mathrm{V}$ & $\frac{\text { Jacanidae }}{\text { Pheasant-tailed Jacana }}$ & Hydrophasianus chirurgus & C & Res, $\mathrm{Br}$ \\
\hline Red-headed Vulture & Sarcogyps calvus ${ }^{\star}(178)$ & 0 & LM & & $(358)$ & & \\
\hline Long-billed Vulture & Gyps indicus $^{\star}(182)$ & UC & LM & Bronze-winged Jacana & Metopidius indicus (359) & C & Res, $\mathrm{Br}$ \\
\hline Indian White-backed & Gyps bengalensis* $(185)$ & UC & $\mathrm{LM}, \mathrm{Br} ?$ & \multicolumn{4}{|l|}{$\underline{\text { Charadriidae }}$} \\
\hline Vulture & & & & $\overline{\text { Red-wattled Lapwing }}$ & Vanellus indicus* $(366)$ & C & Res, $\mathrm{Br}$ \\
\hline Egyptian Vulture & Neophron percnopterus* (187) & UC & Res, Br? & Yellow-wattled Lapwing & Vanellus malabaricus ${ }^{\star}(370)$ & $\mathrm{C}$ & Res, $\mathrm{Br}$ \\
\hline Pallid Harrier & Circus macrourus (190) & UC & WM & Little Ringed Plover & Charadrius dubius (380) & C & Res, Br? \\
\hline Pied Harrier & Circus melanoleucos (192) & UC & WM & Kentish Plover & Charadrius alexandrinus (381) & UC & WM \\
\hline Eurasian Marsh Harrier & Circus aeruginosus (193) & UC & WM & \multicolumn{4}{|l|}{ Scolopacidae } \\
\hline $\begin{array}{l}\text { Short-toed Snake-Eagle } \\
\text { Crested Serpent-Eaqle }\end{array}$ & $\begin{array}{l}\text { Circaetus gallicus (195) } \\
\text { Spilornis cheela (196) }\end{array}$ & $\begin{array}{l}\mathrm{C} \\
\mathrm{uC}\end{array}$ & $\mathrm{Br}$ & Spotted Redshank & Tringa erythropus (392) & 0 & WM \\
\hline Osprey & $\begin{array}{l}\text { Spliornis cneela (196) } \\
\text { Pandion haliaetus (203) }\end{array}$ & UC & Res? & Common Redshank & Tringa totanus (393) & UC & WM \\
\hline & 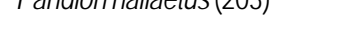 & & & Marsh Sandpiper & Tringa stagnatilis* (395) & $\mathrm{R}$ & WM \\
\hline$\underline{\text { Falconidae }}$ & & & & Common Green Shank & Tringa nebularia (396) & UC & WM \\
\hline Lagger Falcon & Falco jugger (208) & 0 & SLM & Green Sandpiper & Tringa ochropus (397) & UC & WM \\
\hline Peregrine Falcon & Falco peregrinus (211) & 0 & Res? & Wood Sandpiper & Tringa glareola (398) & UC & WM \\
\hline Red-headed Falcon & Falco chicquera (219) & 0 & WM & Common Sandpiper & Tringa actitis* (401) & C & WM \\
\hline Common Kestrel & Falco tinnunculus (224) & C & WM & Wood Snipe & Gallinago nemoricola (405) & $\mathrm{R}$ & $\mathrm{V}$ \\
\hline Galliformes & & & & Pin-tailed Snipe & Gallinago stenura (406) & UC & WM \\
\hline Phasianidae & & & & Common Snipe & Gallinago gallinago (409) & UC & WM \\
\hline$\overline{\text { Painted Francolin }}$ & Francolinus pictus ${ }^{\star}(241)$ & $\mathrm{C}$ & Res, $\mathrm{Br}$ & Jack Snipe & Limnocryptes minimus (410) & $\mathrm{R}$ & V \\
\hline Grey Francolin & $\begin{array}{l}\text { Francolinus pondicerianus }{ }^{*} \\
(246)\end{array}$ & C & Res, $\mathrm{Br}$ & $\begin{array}{l}\text { Little Stint } \\
\text { Temminck's Stint }\end{array}$ & $\begin{array}{l}\text { Calidris minuta (416) } \\
\text { Calidris temminckii (417) }\end{array}$ & $\begin{array}{l}\mathrm{C} \\
\mathrm{UC}\end{array}$ & $\begin{array}{l}\text { WM } \\
\text { WM }\end{array}$ \\
\hline Common Quail & Coturnix coturnix (250) & UC & Res, Br? & Black-winged Stilt & Himantopus himantopus (430) & C & SLM \\
\hline Rain Quail & Coturnix coromandelica (252) & $\mathrm{C}$ & Res, $\mathrm{Br}$ & Pied Avocet & Recurvirostra avosetta (432) & $\mathrm{R}$ & V \\
\hline Blue-breasted Quail & Coturnix chinensis (253) & UC & Res, Br? & \multicolumn{4}{|l|}{ Burhinidae } \\
\hline Jungle Bush-Quail & Perdicula asiatica (255) & C & Res, $\mathrm{Br}$ & Stone Curlew & Burhinus oedicnemus (436) & C & $\mathrm{Res}, \mathrm{Br}$ \\
\hline Rock Bush-Quail & Perdicula argoondah (260) & UC & Res, Br? & Great Thick-knee & Esacus recurvirostris (437) & UC & Res? \\
\hline Red Spurfowl & Galloperdix spadicea* (275) & C & Res, $\mathrm{Br}$ & \multirow{2}{*}{\multicolumn{4}{|c|}{$\underline{\text { Glareolidae }}$}} \\
\hline Painted Spurfowl & Galloperdix lunulata (278) & UC & Res, $\mathrm{Br}$ & & & & \\
\hline Grey Junglefowl & Gallus sonneratii* (301) & C & Res, $\mathrm{Br}$ & Indian Courser & $\begin{array}{l}\text { Cursorius coromandelicus } \\
(440)\end{array}$ & $\mathrm{C}$ & Res, B? \\
\hline Indian Peafowl & Pavo cristatus ${ }^{\star}(311)$ & C & Res, $\mathrm{Br}$ & Small Pratincole & $\begin{array}{l}(440) \\
\text { Glareola lactea (444) }\end{array}$ & UC & Res \\
\hline $\begin{array}{l}\text { Gruiformes } \\
\text { Turnicidae }\end{array}$ & & & & \multicolumn{4}{|l|}{ Laridae } \\
\hline Small Bustardquail & Turnix sylvatica (313) & C & $\mathrm{Res}, \mathrm{Br}$ & Brown-headed Gull & Larus brunnicephalus (454) & UC & WM \\
\hline Yellow-legged Buttonquail & Turnix tanki (314) & UC & $\mathrm{Res}, \mathrm{Br}$ & $\begin{array}{l}\text { Whiskered Tern } \\
\text { River Tern }\end{array}$ & $\begin{array}{l}\text { Chlidonias hybridus (458) } \\
\text { Sterna aurantia (463) }\end{array}$ & $\begin{array}{l}\mathrm{C} \\
\mathrm{C}\end{array}$ & $\begin{array}{l}\text { WM } \\
\text { Res, Br? }\end{array}$ \\
\hline Barred Bustardquail & Turnix suscitator* (318) & C & Res, $\mathrm{Br}$ & Black-bellied Tern & Sterna acuticauda (470) & UC & SLM \\
\hline Gruidae & & & & \multicolumn{4}{|l|}{ Columbiformes } \\
\hline Common Crane & Grus grus (320) & $\mathrm{R}$ & V & $\frac{\text { Pteroclididae }}{\text { Indian Sandgrouse }}$ & s exustus & & \\
\hline & & & & Painted Sandgrouse & Pterocles indicus (492) & UC & SLM \\
\hline
\end{tabular}




\begin{tabular}{|c|c|c|c|c|c|c|c|}
\hline Common name & Scientific name & Abn & Status & Common name & Scientific name & Abn & Status \\
\hline$\underline{\text { Columbidae }}$ & & & & Asian Palm-Swift & Cypsiurus balasiensis (707) & C & $\mathrm{Res}, \mathrm{Br}$ \\
\hline Yellow-legged Green & Treron phoenicoptera* (504) & C & Res, $\mathrm{Br}$ & Hemiprocnidae & & & \\
\hline $\begin{array}{l}\text { Pigeon } \\
\text { Green Imnerial-Pigeon }\end{array}$ & Ducula aenea* (507) & 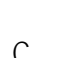 & $\operatorname{Res} \mathrm{Br}$ & $\overline{\text { Crested Tree-swift }}$ & Hemiprocne coronata (709) & $\mathrm{C}$ & $\mathrm{Res}, \mathrm{Br}$ \\
\hline $\begin{array}{l}\text { Green Imperial-Pigeon } \\
\text { Rock Pigeon }\end{array}$ & $\begin{array}{l}\text { Ducula aenea*(507) } \\
\text { Columba livia* }(517)\end{array}$ & C & Res, $\mathrm{Br}$ & Coraciiformes & & & \\
\hline Oriental Turtle Dove & Streptopelia orientalis (533) & UC & Res, Br? & $\underline{\text { Alcedinidae }}$ & & & \\
\hline Eurasian Collared-Dove & Streptopelia decaocto* $(534)$ & $\mathrm{C}$ & Res, $\mathrm{Br}$ & Small Blue Kingfisher & Alcedo atthis (723) & C & Res, $\mathrm{Br}$ \\
\hline Red Collared-Dove & $\begin{array}{l}\text { Streptopelia tranquebarica } \\
\text { (535) }\end{array}$ & C & Res, $\mathrm{Br}$ & $\frac{\text { Cerylidae }}{\text { LescerPied Kinaficher }}$ & & & \\
\hline Spotted Dove & Streptopelia chinensis* (537) & $\mathrm{C}$ & Res, $\mathrm{Br}$ & Lesser Pled KIngtisner & Ceryle rudıs (719) & $\mathrm{C}$ & Res, $\mathrm{Br}$ \\
\hline Laughing Dove & $\begin{array}{l}\text { Streptopelia senegalensis* } \\
\text { (541) }\end{array}$ & C & Res, $\mathrm{Br}$ & 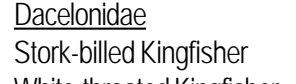 & Halcyon capensis (730) & UC & Res, Br? \\
\hline Emerald Dove & Chalcophaps indica (542) & C & Res, $\mathrm{Br}$ & White-throated Kingfisher & Halcyon smyrnensis (735) & C & Res, Br \\
\hline Psittaciformes & & & & Meropidae & & & \\
\hline Psittacidae & & & & Chestnutheaded & Merops leschenaultii (744) & UC & Res, Br? \\
\hline Alexandrine Parakeet & Psittacula eupatria* (545) & UC & Res, Br? & Bee-eater & & & \\
\hline Rose-ringed Parakeet & Psittacula krameri` $(550)$ & C & Res, $\mathrm{Br}$ & Blue-tailed Bee-eater & Merops philippinus (748) & UC & WM \\
\hline $\begin{array}{l}\text { Plum-headed } \\
\text { Parakeet }\end{array}$ & $\begin{array}{l}\text { Psittacula cyanocepahala* } \\
\text { (558) }\end{array}$ & C & $\mathrm{Res}, \mathrm{Br}$ & $\begin{array}{l}\text { Green Bee-eater } \\
\text { Blue-bearded Bee-eater }\end{array}$ & $\begin{array}{l}\text { Merops orientalis* }(750) \\
\text { Nyctyornis athertoni }(753)\end{array}$ & $\begin{array}{l}\mathrm{C} \\
\mathrm{UC}\end{array}$ & $\begin{array}{l}\text { Res, } \mathrm{Br} \\
\text { Res, } \mathrm{Br} \text { ? }\end{array}$ \\
\hline Cuculiformes & & & & Coraciidae & & & \\
\hline$\underline{\text { Cuculidae }}$ & & & & Indian Roller & Coracias benghalensis* (755) & C & Res, $\mathrm{Br}$ \\
\hline Pied Cuckoo & Clamator jacobinus* $(570)$ & C & Res, Br? & Upupidae & & & \\
\hline Indian Hawk-Cuckoo & Hierococcyx varius* $(573)$ & C & Res, $\mathrm{Br}$ & Common Hoopoe & Upupa epops ${ }^{\star}(763)$ & C & $\mathrm{Res}, \mathrm{Br}$ \\
\hline Indian Cuckoo & Cuculus micropterus (576) & UC & Res, Br? & Bucerotridae & & & \\
\hline $\begin{array}{l}\text { Grey-bellied Cuckoo } \\
\text { Asian Koel }\end{array}$ & $\begin{array}{l}\text { Cacomantis passerinus }{ }^{\star}(584) \\
\text { Eudvnamys scolopacea* }(590)\end{array}$ & $\begin{array}{l}\text { UC } \\
\text { C }\end{array}$ & $\begin{array}{l}\text { Res, } \mathrm{Br} \\
\text { Res, } \mathrm{Br}\end{array}$ & Indian Grey Hornbill & Ocyceros birostris* $(767)$ & C & $\mathrm{Res}, \mathrm{Br}$ \\
\hline Blue-faced Malkoha & $\begin{array}{l}\text { Phaenicophaeus viridirostris* } \\
\text { (595) }\end{array}$ & C & Res, $\mathrm{Br}$ & $\begin{array}{l}\text { Indian Pied Hornbill } \\
\text { Piciformes }\end{array}$ & Anthracoceros coronatus (775) & ) $R$ & Res? \\
\hline Sirkeer Malkoha & $\begin{array}{l}\text { Phaenicophaeus leshenaultii } \\
\text { (598) }\end{array}$ & UC & Res, $\mathrm{Br}$ & $\frac{\text { Capitonidae }}{\text { Brown-headed Barbet }}$ & Megalaima zeylanica* (780) & C & $\mathrm{Res}, \mathrm{Br}$ \\
\hline $\begin{array}{l}\text { Greater Coucal } \\
\text { Strigiformes }\end{array}$ & Centropus sinensis* $(602)$ & C & Res, $\mathrm{Br}$ & Coppersmith Barbet & $\begin{array}{l}\text { Megalaima haemacephala* } \\
\text { (792) }\end{array}$ & C & Res, Br \\
\hline Strigidae & & & & Picidae & & & \\
\hline Eurasian Collared & Otus bakkamoena* (622) & UC & Res, Br? & Eurasian Wryneck & Jynx torquilla (796) & UC & WM \\
\hline Scops-Owl & & & & Streak-throated & Picus xanthopygaeus (808) & UC & Res? \\
\hline Eagle-Owl & Bubo bubo (627) & C & Res, Br & Woodpecker & & & \\
\hline Brown Fish-Owl & Ketupa zeylonensis (631) & UC & Res, $\mathrm{Br}$ & Lesser Yellow-naped & Picus chlorolophus (816) & UC & Res, Br? \\
\hline Jungle Owlet & Glaucidium radiatum* (636) & C & Res, $\mathrm{Br}$ & Woodpecker & & & \\
\hline Brown Hawk-Owl & Ninox scutulata (642) & UC & Res, Br? & Black-rumped Flame- & Dinopium benghalense* (819) & C & Res, $\mathrm{Br}$ \\
\hline Spotted Owlet & Athene brama* $(652)$ & C & $\mathrm{Res}, \mathrm{Br}$ & backed Woodpecker & & & \\
\hline Mottled Wood-Owl & Strix ocellata (657) & UC & Res, Br? & Yellow-crowned & Dendrocopos mahrattensis* & C & Res, $\mathrm{Br}$ \\
\hline Brown Wood-Owl & Strix leptogrammica (660) & UC & Res, Br? & Woodpecker & $(847)$ & & \\
\hline Short-eared Owl & Asio flammeus (664) & $\mathrm{R}$ & WM & $\begin{array}{l}\text { Brown-capped } \\
\text { Woodpecker }\end{array}$ & Dendrocopos nanus* (852) & C & Res, $\mathrm{Br}$ \\
\hline$\frac{\text { Tytonidae }}{\text { Barn Owl }}$ & Tyto alba (606) & UC & Res, Br? & $\begin{array}{l}\text { Black-shouldered } \\
\text { Woodpecker }\end{array}$ & Chrysocolaptes festivus (858) & UC & Res, $\mathrm{Br}$ ? \\
\hline $\begin{array}{l}\text { Caprimulgiformes } \\
\text { Caprimulgidae }\end{array}$ & & & & Passeriformes & & & \\
\hline Grey Nightjar & Caprimulgus indicus (671) & C & Res, $\mathrm{Br}$ & $\frac{\text { Pitddae }}{\text { Indian Pitta }}$ & Pitta hrachvura* (867) & $U C$ & WM \\
\hline $\begin{array}{l}\text { Jerdon's Nightjar } \\
\text { Indian Nightjar }\end{array}$ & $\begin{array}{l}\text { Caprimulgus atripennis (676) } \\
\text { Caprimulqus asiaticus* }(680)\end{array}$ & $\begin{array}{l}\mathrm{O} \\
\mathrm{C}\end{array}$ & $\begin{array}{l}\text { Res? } \\
\text { Res, Br }\end{array}$ & Alaudidae & 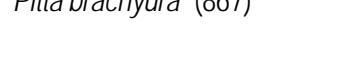 & C & VVIVI \\
\hline Anodiformes & & & & $\overline{\text { Madras Bush Lark }}$ & Mirafra affinis* $(874)$ & UC & Res, $\mathrm{Br}$ \\
\hline Apodidae & & & & Red-winged Bush-Lark & Mirafra erythroptera (877) & UC & Res, Br? \\
\hline Alpine Swift & Tachymarptis melba (693) & UC & SLM & Ashy-crowned Sparrow- & Eremopterix grisea* (878) & $\mathrm{C}$ & Res, $\mathrm{Br}$ \\
\hline House Swift & Apus affinis* (703) & $\mathrm{C}$ & $\mathrm{Res}, \mathrm{Br}$ & & & & \\
\hline
\end{tabular}




\begin{tabular}{|c|c|c|c|c|c|c|c|}
\hline Common name & Scientific name & Abn & Status & Common name & Scientific name & Abn & Status \\
\hline Rufous-tailed Lark & $\begin{array}{l}\text { Ammomanes phoenicurus } \\
\text { (882) }\end{array}$ & C & $\mathrm{Res}, \mathrm{Br}$ & $\frac{\text { Pycnonotidae }}{\text { Red-whiskered Bulbul }}$ & Pycnonotus jacosus (1121) & 0 & SLM \\
\hline Greater Short-toed Lark & Calandrella brachydactyla (886) & $R$ & WM & Red-vented Bulbul & Pycnonotus cafer* (1128) & C & Res, Br \\
\hline $\begin{array}{l}\text { Sykes's Lark } \\
\text { Eastern Skylark }\end{array}$ & $\begin{array}{l}\text { Galerida deva (902) } \\
\text { Alauda gulgula (907) }\end{array}$ & $\begin{array}{l}\mathrm{C} \\
\mathrm{C}\end{array}$ & $\begin{array}{l}\text { Res, } \mathrm{Br} \\
\text { Res, } \mathrm{Br}\end{array}$ & Yellow-throated Bulbul & $\begin{array}{l}\text { Pycnonotus xantholaemus } \\
\text { (1135) }\end{array}$ & 0 & Res, Br? \\
\hline$\underline{\text { Hirundinidae }}$ & & & & White-browed Bulbul & Pycnonotus luteolus (1138) & C & Res, $\mathrm{Br}$ \\
\hline Dusky Crag-Martin & Hirundo concolor (914) & C & Res? & Yellow-browed Bulbul & Iole indica (1144) & $\mathrm{R}$ & \\
\hline Barn Swallow & Hirundo rustica* (916) & $\mathrm{C}$ & WM & $\underline{\text { Cisticolidae }}$ & & & \\
\hline Wire-tailed Swallow & Hirundo smithii (921) & UC & SLM & Zitting Cisticola & Cisticola juncidis* (1498) & $\mathrm{C}$ & Res, $\mathrm{Br}$ \\
\hline Streak-throated Swallow & Hirundo fluvicola (922) & UC & SLM & Grey-breasted Prinia & Prinia hodgsonii (1503) & $\mathrm{C}$ & Res, $\mathrm{Br}$ \\
\hline Red-rumped Swallow & Hirundo daurica* $(927)$ & C & $\mathrm{Res}, \mathrm{Br}$ & Plain Prinia & Prinia inornata ${ }^{\star}(1511)$ & C & Res, $\mathrm{Br}$ \\
\hline Laniidae & & & & Ashy Prinia & Prinia socialis* ${ }^{\star}(1517)$ & C & $\mathrm{Res}, \mathrm{Br}$ \\
\hline Southern Grey Shrike & Lanius meridionalis* (933) & UC & Res? & Jungle Prinia & Prinia sylvatica (1521) & C & $\mathrm{Res}, \mathrm{Br}$ \\
\hline Bay-backed Shrike & Lanius vittatus* $(940)$ & $\mathrm{C}$ & $\mathrm{Res}, \mathrm{Br}$ & $\underline{\text { Muscicapidae }}$ & & & \\
\hline Long-tailed Shrike & Lanius schach ${ }^{\star}(947)$ & $\mathrm{C}$ & $\mathrm{Res}, \mathrm{Br}$ & Spotted Babbler & Pellorneum ruficeps (1154) & UC & Res, $\mathrm{Br}$ \\
\hline Brown Shrike & Lanius cristatus (949) & UC & WM & Indian Scimitar Babbler & $\begin{array}{l}\text { Pomatorhinus horsfieldiï } \\
\text { (1173) }\end{array}$ & C & Res, $\mathrm{Br}$ \\
\hline$\frac{\text { Sturnidae }}{\text { Chestnut-tailed Starling }}$ & & UC. & & Rufous-bellied Babbler & Dumetia hyperythra* (1222) & $\mathrm{C}$ & $\mathrm{Res}, \mathrm{Br}$ \\
\hline Brahminy Starling & Sturnus pagodarum* $(994)$ & $\mathrm{C}$ & $\begin{array}{l}\text { WVIVI } \\
\text { Res, }\end{array}$ & Yellow-eyed Babbler & Chrysomma sinense ${ }^{*}(1231)$ & C & $\mathrm{Res}, \mathrm{Br}$ \\
\hline Rosy Starling & Sturnus roseus (996) & $\mathrm{C}$ & WM & Common Babbler & Turdoides caudatus* (1254) & C & Res, $\mathrm{Br}$ \\
\hline Common Starling & Sturnus vulgaris (997) & 0 & WM & Large Grey Babbler & Turdoides malcolmi* (1258) & $\mathrm{C}$ & Res, $\mathrm{Br}$ \\
\hline Common Myna & Acridotheres tristis* (1006) & $\mathrm{C}$ & $\mathrm{Res}, \mathrm{Br}$ & Jungle Babbler & Turdoides striatus* $(1262)$ & C & Res, $\mathrm{Br}$ \\
\hline Jungle Myna & Acridotheres fuscus (1010) & 0 & Res? & White-headed Babbler & Turdoides affinis* $(1267)$ & C & Res, $\mathrm{Br}$ \\
\hline Corvidae & & & & Quaker Babbler & Alcippe poioicephala (1389) & UC & Res? \\
\hline Southeastern Treepie & Dendrocitta vaqabunda* (1034) & & & Brown Flycatcher & Muscicapa dauurica*(1407) & UC & WM \\
\hline House Crow & Corvus splendens* (1049) & $c$ & $\mathrm{Res}, \mathrm{Br}$ & Brown-breasted & Muscicapa muttui (1408) & 0 & WM \\
\hline Jungle Crow & Corvus macrorhynchos ${ }^{\star}(1057)$ & & & Flycatcher & & & \\
\hline Eurasian Golc & & $\mathrm{C}$ & $\mathrm{Res}, \mathrm{Br}$ & Red-breasted Flycatcher & Ficedula parva*(1411) & UC & WM \\
\hline Black-naped Oriole & Oriolus chinensis (954) & C & $\mathrm{Res}, \mathrm{Br}$ & Little Pied Flycatcher & Ficedula westermanni (1419) & $\mathrm{R}$ & V \\
\hline Black-hooded Oriole & Oriolus xanthornus* (959) & $\begin{array}{l}R \\
C\end{array}$ & SLIVI & & & & \\
\hline Black Drongo & Dicrurus macrocercus* $(963)$ & C & Res, $\mathrm{Br}$ & $\begin{array}{l}\text { While-browed Blue } \\
\text { Flycatcher }\end{array}$ & Muscicapa superciliaris (1421) & 0 & WM \\
\hline Ashy Drongo & Dicrurus leucophaeus* $(965)$ & UC & WM & Tickell's Blue Flycatcher & Cyornis tickelliae* ${ }^{\star}(1442)$ & C & Res, $\mathrm{Br}$ \\
\hline White-bellied Drongo & Dicrurus caerulescens ${ }^{\star}(967)$ & C & $\mathrm{Res}, \mathrm{Br}$ & Verditer Flycatcher & Eumyias thalassina (1445) & UC & WM \\
\hline Bronzed Drongo & Dicrurus aeneus (971) & UC & Res, Br? & Grey-headed Flycatcher & Culicicapa ceylonensis (1449) & UC & WM \\
\hline Spangled Drongo & Dicrurus hottentottus (973) & UC & Res, Br? & White-browed Fantail & Rhipidura aureola* (1452) & $\mathrm{C}$ & Res, $\mathrm{Br}$ \\
\hline Greater Racket-tailed & Dicrurus paradiseus (976) & UC & SLM & Flycatcher & & & \\
\hline Drongo & & & & White-throated Fantail & Rhipidura albicollis* (1458) & UC & Res, Br? \\
\hline Ashy Wood-swallow & Artamus fuscus* (982) & C & Res, Br? & Flycatcher & & & \\
\hline Lesser Wood Shrike & $\begin{array}{l}\text { Tephrodornis pondicerianus* } \\
\text { (1070) }\end{array}$ & C & $\mathrm{Res}, \mathrm{Br}$ & $\begin{array}{l}\text { Paradise Flycatcher } \\
\text { Black-naped Flycatcher }\end{array}$ & $\begin{array}{l}\text { Terpsiphone paradisi` }(1461) \\
\text { Hypothymis azurea }(1465)\end{array}$ & $\begin{array}{l}\mathrm{C} \\
\mathrm{UC}\end{array}$ & $\begin{array}{l}\text { Res, } \mathrm{Br} \\
\text { Res, } \mathrm{Br}\end{array}$ \\
\hline Large Cuckoo-Shrike & Coracina macei* ${ }^{\star}(1072)$ & $\mathrm{C}$ & $\mathrm{Res}, \mathrm{Br}$ & Bluethroat & Luscinia svecicus (1644) & 0 & WM \\
\hline Black-headed & Coracina melanoptera* $(1079)$ & C & $\mathrm{Res}, \mathrm{Br}$ & Oriental Magpie-Robin & Copsychus saularis* (1661) & $\mathrm{C}$ & Res, $\mathrm{Br}$ \\
\hline Cuckoo-shrike & & & & White-rumped Shama & Copsychus malabaricus (1665) & 0 & Res? \\
\hline Scarlet Minivet & Pericrocotus flammeus (1081) & 0 & SLM & Black Redstart & Phoenicurus ochruros* (1672) & UC & WM \\
\hline Small Minivet & $\begin{array}{l}\text { Pericrocotus cinnamomeus* } \\
\text { (1093) }\end{array}$ & $\mathrm{C}$ & $\mathrm{Res}, \mathrm{Br}$ & $\begin{array}{l}\text { Common Stone-chat } \\
\text { Pied Bush-chat }\end{array}$ & $\begin{array}{l}\text { Saxicola torquata* (1697) } \\
\text { Saxicola caprata* }(1701)\end{array}$ & $\begin{array}{l}\text { UC } \\
\mathrm{C}\end{array}$ & $\begin{array}{l}\text { WM } \\
\text { Res, Br? }\end{array}$ \\
\hline Whitebellied Minivet & $\begin{array}{l}\text { Pericrocotus erythropygius* } \\
\text { (1096) }\end{array}$ & $\mathrm{O}$ & Res? & $\begin{array}{l}\text { Indian Robin } \\
\text { Blue Rock Thrush }\end{array}$ & $\begin{array}{l}\text { Saxicoloides fulicata* }(1719) \\
\text { Monticola solitarius (1726) }\end{array}$ & $\begin{array}{l}\mathrm{C} \\
\mathrm{UC}\end{array}$ & $\begin{array}{l}\text { Res, Br } \\
\text { WM }\end{array}$ \\
\hline Irenidae & & & & Orange-headed Thrush & Zoothera citrina (1734) & UC & Res, $\mathrm{Br}$ \\
\hline Common lora & Aegithinia tiphia* (1099) & C & $\mathrm{Res}, \mathrm{Br}$ & Eurasian Blackbird & Turdus merula (1753) & UC & SLM \\
\hline Goldfronted Chloropsis & Chloropsis aurifrons (1104) & $\mathrm{C}$ & $\mathrm{Res}, \mathrm{Br}$ & Sylviidae & & & \\
\hline Goldmantled Chloropsis & $\begin{array}{l}\text { Chloropsis cochinchinensis }{ }^{*} \\
\text { (1107) }\end{array}$ & C & $\mathrm{Res}, \mathrm{Br}$ & $\begin{array}{l}\text { Common Tailorbird } \\
\text { Clamorous Reed } \\
\text { Warbler }\end{array}$ & $\begin{array}{l}\text { Orthotomus sutorius }{ }^{\star}(1535) \\
\text { Acrocephalus stentoreus } \\
(1550)\end{array}$ & $\begin{array}{l}C \\
U C\end{array}$ & $\begin{array}{l}\text { Res, Br } \\
\text { WM }\end{array}$ \\
\hline
\end{tabular}




\begin{tabular}{|c|c|c|c|c|c|c|c|}
\hline Common name & Scientific name & Abn & Status & Common name & Scientific name & Abn & Status \\
\hline Blyth's Reed-Warbler & $\begin{array}{l}\text { Acrocephalus dumetorum } \\
\text { (1556) }\end{array}$ & $\mathrm{C}$ & WM & $\begin{array}{l}\text { Citrine Wagtail } \\
\text { Grey Wagtail }\end{array}$ & $\begin{array}{l}\text { Motacilla citreola }(1881) \\
\text { Motacilla cinerea* }(1884)\end{array}$ & $\begin{array}{l}\mathrm{R} \\
\mathrm{UC}\end{array}$ & $\begin{array}{l}\text { WM } \\
\text { WM }\end{array}$ \\
\hline Booted Warbler & Hippolais caligata (1563) & C & WM & White Wagtail & Motacilla alba (1885) & $\mathrm{C}$ & WM \\
\hline $\begin{array}{l}\text { Orphean Warbler } \\
\text { LesserWhitethroat }\end{array}$ & Sylvia hortensis (1565) & 0 & WM & Large Pied Wagtail & $\begin{array}{l}\text { Motacilla maderaspatensis* } \\
\text { (1891) }\end{array}$ & C & Res, $\mathrm{Br}$ \\
\hline $\begin{array}{l}\text { Lesser Whitethroat } \\
\text { Hume's Lesser }\end{array}$ & $\begin{array}{l}\text { Sylvia curruca (156/) } \\
\text { Sylvia (curruca) althaea (1570) }\end{array}$ & 0 & $\begin{array}{l}\text { WM } \\
\text { WM }\end{array}$ & House Sparrow & $\begin{array}{l}\text { (1891) } \\
\text { Passer domesticus* }\end{array}$ & C & Res, $\mathrm{Br}$ \\
\hline $\begin{array}{l}\text { White-throat } \\
\text { Tickell's Leaf-Warbler }\end{array}$ & Phylloscopus affinis (1579) & 0 & WM & $\begin{array}{l}\text { Chestnut-shouldered } \\
\text { Petronia }\end{array}$ & Petronia xanthocollis* (1949) & UC & $\mathrm{Res}, \mathrm{Br}$ \\
\hline Hume's Warbler & Phylloscopus humei (1590) & 0 & WM & Baya Weaver & Ploceus philippinus* (1957) & $\mathrm{C}$ & Res, $\mathrm{Br}$ \\
\hline Large-billed Leaf-Warbler & $\begin{array}{l}\text { Phylloscpus magnirostris* } \\
\text { (1601) }\end{array}$ & UC & WM & $\begin{array}{l}\text { Plain Munia } \\
\text { White-rumped Munia }\end{array}$ & $\begin{array}{l}\text { Lonchura malabarica* }(1966) \\
\text { Lonchura striata }(1968)\end{array}$ & $\begin{array}{l}\mathrm{C} \\
\mathrm{UC}\end{array}$ & $\begin{array}{l}\text { Res, } \mathrm{Br} \\
\text { Res, } \mathrm{Br}\end{array}$ \\
\hline Greenish Warbler & $\begin{array}{l}\text { Phylloscopus trochiloides } \\
\text { (1604) }\end{array}$ & UC & WM & $\begin{array}{l}\text { Scaly-breasted Munia } \\
\text { Black-headed Munia }\end{array}$ & $\begin{array}{l}\text { Lonchura punctulata* }(1974) \\
\text { Lonchura malacca (1978) }\end{array}$ & $\begin{array}{l}\mathrm{C} \\
\mathrm{UC}\end{array}$ & $\begin{array}{l}\text { Res, } \mathrm{Br} \\
\text { Res, } \mathrm{Br}\end{array}$ \\
\hline Western Crowned Leaf & Phylloscopus occipitalis (1606) & 0 & WM & Nectariniidae & & & \\
\hline Warbler & & & & Thick-billed Flowerpecker & Dicaeum agile*(1892) & C & Res, Br \\
\hline$\frac{\text { Paridae }}{\text { Great Tit }}$ & Parus major* (1795) & $\mathrm{C}$ & $\mathrm{Res}, \mathrm{Br}$ & Tickell's Flowerpecker & $\begin{array}{l}\text { Dicaeum erythrorhynchos* } \\
\text { (1899) }\end{array}$ & C & $\mathrm{Res}, \mathrm{Br}$ \\
\hline $\begin{array}{l}\text { Yellow-lored Yellow Tit } \\
\text { Sittidae }\end{array}$ & Parus xanthogenys* $(1810)$ & C & $\mathrm{Res}, \mathrm{Br}$ & $\begin{array}{l}\text { Purple-rumped Sunbird } \\
\text { Loten's Sunbird }\end{array}$ & $\begin{array}{l}\text { Nectarinia zeylonica* }(1907) \\
\text { Nectarinia lotenia }(1911)\end{array}$ & $\begin{array}{l}\mathrm{C} \\
\mathrm{UC}\end{array}$ & $\begin{array}{l}\text { Res, } \mathrm{Br} \\
\text { Res, Br? }\end{array}$ \\
\hline Velvet-fronted Nuthatch & Sitta frontalis* (1838) & UC & Res, Br? & Purple Sunbird & Nectarinia asiatica* (1917) & C & Res, $\mathrm{Br}$ \\
\hline$\frac{\text { Passeridae }}{\text { Olive-backed Pipit }}$ & Anthus hodgsoni (1852) & 0 & WM & $\frac{\text { Zosteropidae }}{\text { Oriental White-eye }}$ & Zosterops palpebrosus* $(1934)$ & $\mathrm{C}$ & Res, $\mathrm{Br}$ \\
\hline Tree Pipit & Anthus trivialis (1854) & UC & WM & & & & \\
\hline Paddyfield Pipit & Anthus rufulus (1859) & UC & Res, Br? & \multirow{5}{*}{\multicolumn{4}{|c|}{$\begin{array}{l}\text { Abn- Abundance } \\
\text { C - Common; UC - Uncommon; O - Occasional; R - Rare } \\
\text { Res - Resident; Br - Breeding; SLM - Seasonal Local Migrant; LM - Local } \\
\text { Migrant; WM - Winter Migrant; V - Vagrant; ? - Probable status } \\
\text { * species recorded by Dr. Sàlim Ali during Hyderabad State Ornithological Survey, } \\
\text { 1931-32; numbers in parentheses are Synopsis reference number of Ripley (1982). }\end{array}$}} \\
\hline Tawny Pipit & Anthus campestris* (1861) & 0 & WM & & & & \\
\hline Blyth's Pipit & Anthus godlewskii (1863) & 0 & WM & & & & \\
\hline Forest Wagtail & Dendronanthus indica* (1874) & 0 & WM & & & & \\
\hline Yellow Wagtail & Motacilla flava $(1875,1876)$ & UC & WM & & & & \\
\hline
\end{tabular}

